\section{Das thermische Rauschen eines Dielektrikums mit Verlusten}

\section{JÖrn Petersson}

Institut für Experimentalphysik II

der Universität des Saarlandes, Saarbrücken

(Z. Naturforsch. 25 a, 148-149 [1970] ; eingeg. am 12. November 1969)

Das thermische Rauschen von dielektrischen Stoffen, die bei tiefen Temperaturen ferroelektrisch sind, ist verschiedentlich mit dem Ziel untersucht worden, kritische Polarisationsschwankungen in der Umgebung des Umwandlungspunktes nachzuweisen ${ }^{1-4}$. Die theoretische Deutung dieser Messungen geschieht i. allg. durch eine thermodynamische Theorie ${ }^{5}$. Im Gegensatz dazu werden im folgenden unabhängig von einer speziellen Theorie allgemeine Beziehungen für das totale mittlere Schwankungsquadrat von Ladung und Spannung eines Kondensators, in dem sich ein Dielektrikum mit Verlusten befindet, abgeleitet. Dabei werden nur einige sehr allgemeine Annahmen über die Eigenschaften des Materials gemacht: Das Material sei frei von inneren Quellen, das dielektrische Verhalten werde durch lineare und kausale Beziehungen beschrieben und für die Schwankungen im thermischen Gleichgewicht gelte die bekannte Nyquist-Beziehung ${ }^{6}$. Diese lautet für eine komplexe Impedanz $\mathfrak{R}=1 / \mathfrak{G}$ :

$$
\begin{aligned}
W_{\mathrm{u}} & =4 k T \operatorname{Re}(\mathfrak{R}), \\
W_{\mathrm{i}} & =4 k T \operatorname{Re}(\mathfrak{G}) .
\end{aligned}
$$

Dabei sind $W_{\mathrm{u}}$ und $W_{\mathrm{i}}$ die Spektren der Rauschspannung im Leerlauf und des Rauschstromes im Kurzschluß, $k$ die Boltzmann-Konstante und $T$ die absolute Temperatur.

Wir betrachten nun ein dielektrisches Plättchen der Dicke $d$, auf dem sich Elektroden der Fläche $F$ befinden. Die Richtung der Flächennormalen des Plättchens stimme mit einer kristallographischen Achse überein. Die dielektrischen Eigenschaften des Plättchens werden dann durch die von der Kreisfrequenz $\omega$ abhängige Dielektrizitätskonstante (DK)

$$
\varepsilon^{*}(\omega)=\varepsilon^{\prime}(\omega)-i \varepsilon^{\prime \prime}(\omega)
$$

oder durch den dazu reziproken Dielektrizitätsmodul (DM)

$$
\left(\varepsilon^{*}(\omega)\right)^{-1}=\beta^{*}(\omega)=\beta^{\prime}(\omega)+i \beta^{\prime \prime}(\omega)
$$

in dieser Richtung beschrieben. Die Impedanz läßt sich damit unmittelbar angeben und wegen (1) ist das Spektrum der Rauschspannung im Leerlauf durch den Imaginärteil von $\beta^{*}(\omega)$ gegeben :

$$
W_{\mathrm{u}}(\omega)=4 k T \frac{\beta^{\prime \prime}(\omega)}{\omega} \frac{d}{F} .
$$

Sonderdruckanforderungen an Dr. J. Petersson, Institut für Experimentalphysik II der Universität des Saarlandes, D-6600 Saarbrücken, Universität, Bau 4.

1 J. J. Brophy, in: R. E. Burgess (Herausgeber), Fluctuation Phenomena in Solids, Academic Press, London 1965.

2 J. J. Brophy u. S. L. Weвb, Phys. Rev. 128, 584 [1962].

3 H. Bittel, H. G. Unruh u. G. Hellmiss, Z. Phys. 184, 1 [1965].
Aus (1 a) erhält man das Spektrum des bei Kurzschluß der Elektroden fließenden Rauschstromes. $W_{i}(\omega)$ hängt mit dem Spektrum $W_{q}(\omega)$ des mittleren Schwankungsquadrates der Ladung $Q$ auf den Elektroden durch $W_{i}(\omega)=\omega^{2} W_{q}(\omega)$ zusammen. Daher ist $W_{q}(\omega)$ durch den Imaginärteil von $\varepsilon^{*}(\omega)$ gegeben:

$$
W_{q}(\omega)=4 k T \frac{\varepsilon^{\prime \prime}(\omega)}{\omega} \frac{F}{d} .
$$

Die Relationen (2) und (3) sind formal gleich aufgebaut. Dies ist ein Ausdruck der Tatsache, daß im Sinne der Thermodynamik Ladung und Spannung ein Paar von konjugierten äußeren Variablen zur Beschreibung des Zustandes des Dielektrikums bilden. In (2) ist offenbar die Ladung auf den Elektroden gegeben, während in (3) die Spannung fest ist.

Die Spektren (2) und (3) lassen sich auf die der elektrischen Feldstärke $E$ und der dielektrischen Verschiebung $D$ unter Verwendung des Volumens $V=F d$ umschreiben:

$$
\begin{aligned}
& W_{\mathrm{E}}(\omega)=\frac{4 k T}{V} \frac{\beta^{\prime \prime}(\omega)}{\omega}, \\
& W_{\mathrm{D}}(\omega)=\frac{4 k T}{V} \frac{\varepsilon^{\prime \prime}(\omega)}{\omega} .
\end{aligned}
$$

Um das totale mittlere Schwankungsquadrat der Spannung und der Ladung bzw. von $E$ und $D$ in den beiden Fällen zu erhalten, benutzen wir die Tatsache, daß Real- und Imaginärteil einer Materialgröße nicht unabhängig voneinander sind, da sie die Kramers-KronigRelationen ${ }^{7}$ erfüllen müssen. Eine von ihnen lautet für den Modul $\beta^{*}(\omega)$ :

$$
\beta^{\infty}-\beta^{\prime}(\omega)=\frac{2}{\pi} \int_{0}^{\infty} \frac{u \beta^{\prime \prime}(u)}{u^{2}-\omega^{2}} \mathrm{~d} u .
$$

Dabei ist $\beta^{\infty}$ der reelle frequenzunabhängige DM, gegen den $\beta^{*}(\omega)$ für $\omega \rightarrow \infty$ strebt. Setzt man in (4) $\omega=0$, so läßt sich $\overline{U^{2}}$ unmittelbar angeben. Man erhält, wenn $\beta^{0}$ den reellen DM für $f=0$ bezeichnet, für die gesamte Rauschspannung im Leerlauf:

$$
\overline{U^{2}}=k T\left(\beta^{\infty}-\beta^{0}\right) \frac{d}{F} .
$$

Aus einer zu (4) analogen Beziehung für $\varepsilon^{*}(\omega)$ ergibt sich entsprechend für die gesamte Ladungsschwankung bei Kurzschluß :

$$
\overline{Q^{2}}=k T\left(\varepsilon^{0}-\varepsilon^{\infty}\right) \frac{F}{d} .
$$

Das totale mittlere Schwankungsquadrat von $U$ bzw. von $E$ im Leerlauf und von $Q$ bzw. $D$ im Kurzschlu $\beta$

4 F. Micheron, C. Baumberger u. L. Godefroy, Proc. Int. Meet. Ferroelectricity, The Institute of Physics of the Czechoslovak Academy of Sciences, Prag 1966, S. 185.

5 R. E. Burgess, Can. J. Phys. 36, 1569 [1958].

${ }^{6}$ H. NyQuist, Phys. Rev. 32, 110 [1928].

7 E. Hiedemann u. R. D. Spence, Z. Phys. 133, 109 [1952]. 
hängt also nur von dem Unterschied zwischen der jeweiligen hoch- und niederfrequenten Materialgröße ab. Im Falle einer Resonanz oder einer Relaxation sind dies die Resonanz- bzw. die Relaxationsstärke. Für die Ableitung von (5) und (6) wurde die Gültigkeit von (1) und der Kramers-Kronig-Relationen vorausgesetzt. Gleichung (1) für das thermische Rauschen gilt bekanntlich dann, wenn sich das passive lineare System im thermischen Gleichgewicht mit der Umgebung befindet und wenn Quanteneffekte noch keine Rolle spielen, d. h. $\hbar \omega \ll k T$ ist. Gleichung (4) gilt unter den folgenden Voraussetzungen: Ladung und Spannung des passiven linearen Systems hängen durch kausale Beziehungen miteinander zusammen, d. h. ist die Ladung (Spannung) bis zu einer Zeit $t_{0}$ Null, so verschwindet die daraus resultierende Spannung (Ladung) für $t \leqq t_{0}$ ebenfalls. Weiter muß vorausgesetzt werden, daß die $\mathrm{zu}$ einer reellen Ladung (Spannung) gehörige Antwortgröße Spannung (Ladung) ebenfalls reell ist. Die Gln. (5) und (6) gelten somit unabhängig davon, welcher physikalische Mechanismus für die jeweilige Frequenzabhängigkeit der Materialgröße verantwortlich ist.

Bei einer Anwendung von (2), (3), (5) und (6) auf ferroelektrische Phasenübergänge wird man sich auf solche Systeme beschränken, bei denen die ferroelektri-

8 H. E. Müser u. J. Pottharst, Phys. Stat. Sol. 24, 109 [1967].

9 F. SANDy u. R. V. Jones, Phys. Rev. 168, 481 [1968]. sche Dispersionsstufe bei so tiefen Frequenzen liegt, daß man (1) in dem gesamten Bereich des Abfalls voraussetzen kann. Dies ist bei Zimmertemperatur für solche Stoffe der Fall, bei denen diese Dispersion im Bereich der Mikrowellen liegt und oft durch eine Relaxation vom Debye-Typ dargestellt werden kann ${ }^{8-11}$. $\mathrm{Da} \varepsilon^{\infty}$ in der Größenordnung 10 liegt, erkennt man aus (5) und (6), daß das Schwankungsquadrat der Spannung im Leerlauf an einem Umwandlungspunkt zweiter Ordnung endlich bleibt, während das Schwankungsquadrat der Ladung im Kurzschluß sehr groß wird, da $\beta^{0}=1 / \varepsilon^{0} \rightarrow 0$ für $T \rightarrow T_{\text {c }}$. Allerdings setzt diese Betrachtung voraus, daß das Dielektrikum auch in der Umgebung von $T_{\mathrm{c}}$ durch lineare Beziehungen dargestellt werden kann.

Das Verhalten der Spektren (2) und (3) in der Umgebung des Umwandlungspunktes läßt sich allgemein nur angeben, wenn man zusätzliche Annahmen über das Material macht. So kann man zu einer Beschreibung dieser Spektren den Formalismus der Thermodynamik irreversibler Prozesse heranziehen. Je nach den äußeren Randbedingungen lassen sich dann die Schwankungen der äußeren Variablen $D$ oder $E$ auf die Schwankungen der Polarisation, die als innere Variable aufgefaßt werden kann, zurückführen ${ }^{12}$.

10 G. Luther u. H.E. Müser, Z. Naturforsch. 24 a, 389 [1969].

11 I. HatTA, J. Phys. Soc. Japan 24, 1043 [1968].

12 J. Petersson, erscheint 1970 in Z. angew. Phys. 